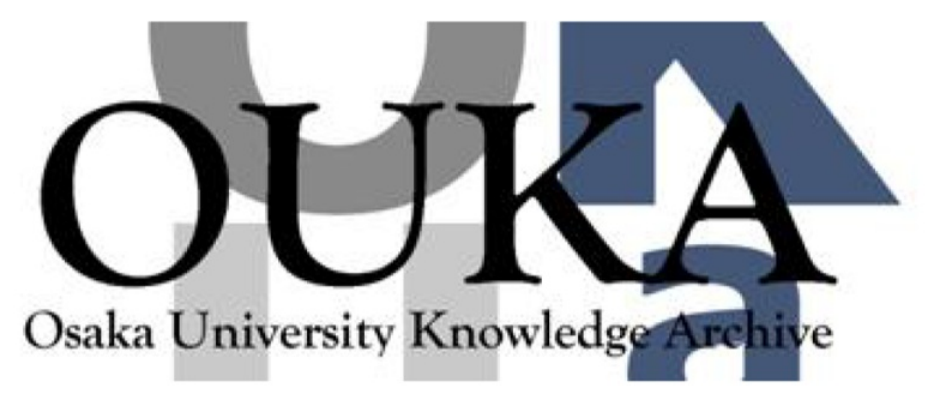

\begin{tabular}{|c|l|}
\hline Title & $\begin{array}{l}\text { Neutrino masses in the supersymmetric standard } \\
\text { model with right-handed neutrinos and } \\
\text { spontaneous R-parity violation }\end{array}$ \\
\hline Author(s) & Kitano, Ryuichiro; 0da, Kinya \\
\hline Citation & Physical Review D. 61(11) p. 113001 \\
\hline Issue Date & $2000-04-25$ \\
\hline oaire:version & VoR \\
\hline URL & https://hdl.handle. net/11094/78781 \\
\hline rights & ○ 2000 American Physical Society \\
\hline Note & \\
\hline
\end{tabular}

Osaka University Knowledge Archive : OUKA

https://ir. Library. osaka-u. ac. jp/

Osaka University 


\title{
Neutrino masses in the supersymmetric standard model with right-handed neutrinos and spontaneous $\boldsymbol{R}$-parity violation
}

\author{
Ryuichiro Kitano \\ Theory Group, KEK, Oho 1-1, Tsukuba, Ibaraki 305-0801, Japan \\ and Department of Particle and Nuclear Physics, The Graduate University for Advanced Studies, Oho 1-1, Tsukuba, \\ Ibaraki 305-0801, Japan \\ Kin-ya Oda \\ Theory Group, KEK, Oho 1-1, Tsukuba, Ibaraki 305-0801, Japan \\ and Department of Physics, Osaka University, Toyonaka, Osaka 560 Japan
}

(Received 12 November 1999; published 25 April 2000)

\begin{abstract}
We propose an extension of the supersymmetric standard model with right-handed neutrinos and a singlet Higgs field, and study the neutrino masses in this model. The Majorana masses for the right-handed neutrinos are generated around the supersymmetry breaking scale through the vacuum expectation value of the singlet Higgs field. This model may induce spontaneous $R$-parity violation via the vacuum expectation value of the right-handed sneutrino. In the case, the effective theory is similar to a bilinear $R$-parity violating model. There are two sources for the neutrino masses: one is this bilinear $R$-parity breaking effect, and the other is the ordinary seesaw effect between left- and right-handed neutrinos. Combining these two effects, the hierarchical neutrino mass pattern arises even when the neutrino Yukawa matrices are not hierarchical. We acquire appropriate masses and mixings to explain both the solar and atmospheric neutrino oscillations.

PACS number(s): 14.60.St, 11.30.Qc, 12.60.Jv, 14.60.Pq
\end{abstract}

\section{INTRODUCTION}

Recent Super-Kamiokande data provide convincing evidence for neutrino oscillations [1]. It strongly suggests the existence of neutrino masses of at most a few eV. The standard explanation of such tiny neutrino masses is the seesaw mechanism [2], in which one introduces right-handed singlet neutrinos $N_{i}$. The standard model gauge group does not forbid the Majorana masses $M_{R}$ between the right-handed neutrinos $N_{i}$, which are usually placed near the unification scale, say $M_{R} \sim 10^{14} \mathrm{GeV}$.

Bilinear $R$-parity violation in the supersymmetric model is another possible way of obtaining the neutrino masses [3]. This model is the extension of the minimal supersymmetric standard model (MSSM) [4] adding small bilinear terms $L_{i} H_{2}$ to the superpotential. Originally the conservation of $R$ parity was introduced to avoid proton decay, so that lepton and baryon number are conserved in the MSSM. The $R$-parity breaking terms $L_{i} H_{2}$ violate lepton number conservation, leaving baryon number conserved. Therefore, neutrino masses are generated without introducing any righthanded neutrinos. In this scenario, the neutrino masses have a characteristic structure. Only one generation of neutrino obtains mass at the tree level by mixing with the gauginos (we refer to this as "gaugino seesaw"). Therefore, the masses of the first and second generation of neutrinos are generated by radiative corrections. In this model it is rather difficult to maintain the second generation neutrino mass appropriately large to account for the solar neutrino anomaly by the Mikheyev-Smirnov-Wolfenstein (MSW) effect.

The origin of the $R$-parity violation may be naturally explained by spontaneous $R$-parity breaking $[5,6]$. However, it is not very easy to generate spontaneous symmetry breaking in the supersymmetric model in general. Namely, the origin [a point where all the vacuum expectation values (VEV's) are zero] is always the global minimum of the scalar potential in the supersymmetric limit, and if we add the positive soft scalar mass-squared terms it still remains at least a local minimum and in general tends to be the global minimum. (We recall that negative soft mass-squared terms for the charged-colored fields easily lead to unwanted charge or color breaking.) One way to get rid of this vacuum is radiative breaking [7], where the radiative correction to the soft scalar mass-squared drives it negative even if it is positive at the tree level. In this mechanism the origin does not remain the local minimum. This is applied to the electroweak symmetry breaking of the MSSM successfully. Another way is to introduce the large soft scalar trilinear couplings ( $A$ terms) [8]. The origin remains a local minimum while there appears the global minimum which is far from the origin due to the effect of the large $A$ terms.

As mentioned above, when one considers the seesaw mechanism the right-handed Majorana masses $M_{R}$ are supposed to lie near the unification scale. This is based on the assumption that at least one of the Dirac-type Yukawa couplings $f^{\nu}$ is of order unity. However, there are no reason $a$ priori to expect $f^{\nu} \sim O(1)$. In fact, almost all the Yukawa couplings in the standard model or the MSSM are much smaller than 1; the top Yukawa coupling is the only exception. ${ }^{1}$ Therefore, it is interesting to pursue the possibility that the right-handed Majorana mass scale $M_{R}$ is also low enough to be spontaneously generated at the weak or supersymmetry breaking scale, while the Dirac-type Yukawa couplings $f^{\nu}$ are small enough to give appropriate seesaw masses of the left-handed neutrinos $\nu$.

\footnotetext{
${ }^{1}$ Also bottom and tau Yukawa couplings become $O(1)$ in the large $\tan \beta\left(v_{1} \ll v_{2}\right)$ region of the MSSM.
} 
In this paper we propose a model where a singlet Higgs field $S$ is added to the MSSM with right-handed neutrinos $N_{i}$. We consider the most general superpotential consistent with $R$ parity and an overall $Z_{3}$ symmetry which forbids an explicit $\mu H_{1} H_{2}$ term, without assuming any extra global symmetry. In particular, we do not impose lepton number symmetry by hand, so that the singlet $S$ may couple to ordinary Higgs fields $H_{1}$ and $H_{2}$, which means that the $\mu$ term is spontaneously generated as well [9].

Our model has an interesting parameter region where leftand right-handed sneutrinos acquire VEV's and $R$ parity is spontaneously broken. In the vacuum with broken $R$ parity, the effective theory is similar to the bilinear $R$ parity violating model. There are two sources for the left-handed neutrino masses $m_{\nu}$ in this case. One is the ordinary seesaw effect with right-handed neutrinos $N_{i}$ and the other is the bilinear $R$-parity breaking effect.

Owing to the existence of these two effects, we can explain the hierarchical neutrino mass structure naturally. The seesaw mechanism with right-handed neutrinos provides masses for all generations, while the effect of the $R$-parity breaking provides mass only for one generation. Therefore, one neutrino may be much heavier than the other two neutrinos.

This paper is organized as follows: In Sec. II, we analyze the scalar potential of our model. In Sec. III, we investigate the neutrino masses with VEV's considered in Sec. II. Sec. IV is the summary of this paper. The detailed calculations of the vacua of the simplified potential are shown in the appendix.

\section{THE MODEL AND ITS VACUA}

We add the singlet field $S$ to the MSSM with right-handed neutrinos $N_{i}$. We assign $R$ parity even for $S$ and odd for $N_{i}$, as usual. The most general superpotential consistent with $R$-parity conservation (up to the appropriate field redefinition) is

$$
W=W_{\mathrm{MSSM}}+W_{N, S}
$$

where

$$
\begin{gathered}
W_{\mathrm{MSSM}}=\sum_{i} f_{i}^{d}\left(H_{1} Q_{i}\right) D_{i}+\sum_{i, j} f_{i j}^{u}\left(Q_{i} H_{2}\right) U_{j} \\
+\sum_{i} f_{i}^{e}\left(H_{1} L_{i}\right) E_{i}, \\
W_{N, S}=\sum_{i, j} f_{i j}^{v}\left(L_{i} H_{2}\right) N_{j}+\lambda_{H}\left(H_{1} H_{2}\right) S+\sum_{i} \frac{\lambda_{N i}}{2} N_{i}^{2} S \\
+\frac{\lambda_{S}}{3 !} S^{3},
\end{gathered}
$$

$i, j$ are generation indices and summed from 1 to 3 . The parameters $f_{i}^{d}, f_{i}^{e}, \lambda_{N i}, \lambda_{H}$, and $\lambda_{S}$ can be taken real and positive while $f_{i j}^{u}$ and $f_{i j}^{\nu}$ are in general complex matrices. We have imposed an overall $Z_{3}$ symmetry in which all the superfields have the same charge, as is done in the next-tominimal supersymmetric standard model to generate the $\mu$ term spontaneously [9]. ${ }^{2}$

The scalar potential corresponding to Eq. (1) is

$$
V=V_{F}+V_{D}+V_{\text {soft }}
$$

where

$$
\begin{gathered}
V_{F}=\sum_{\Phi=D, U, Q}\left|\frac{\partial W_{\mathrm{MSSM}}}{\partial \Phi}\right|^{2}+\sum_{i}\left|f_{i}^{e}\left(H_{1} L_{i}\right)\right|^{2}+\sum_{i}\left|\frac{\partial W_{\mathrm{MSSM}}}{\partial L_{i}}+\sum_{j} f_{i j}^{\nu} H_{2} N_{j}\right|^{2}+\left|\frac{\partial W_{\mathrm{MSSM}}}{\partial H_{1}}+\lambda_{H} H_{2} S\right|^{2} \\
+\left|\frac{\partial W_{\mathrm{MSSM}}}{\partial H_{2}}+\sum_{i, j} f_{i j}^{v} L_{i} N_{j}+\lambda_{H} H_{1} S\right|^{2}+\sum_{j}\left|\sum_{i} f_{i j}^{\nu}\left(L_{i} H_{2}\right)+\lambda_{N j} N_{j} S\right|^{2}+\left|\lambda_{H}\left(H_{1} H_{2}\right)+\sum_{i} \frac{\lambda_{N i}}{2} N_{i}^{2}+\frac{\lambda_{S}}{2} S^{2}\right|^{2} \\
V_{\text {soft }}=V_{\text {soft }}^{\mathrm{MSSM}}+\left(\sum_{i, j} A^{\nu} f_{i j}^{v}\left(L_{i} H_{2}\right) N_{j}+A^{H} \lambda_{H}\left(H_{1} H_{2}\right) S+\text { H.c. }\right) \\
+\left[\sum_{i} m^{2}\left|N_{i}\right|^{2}+m^{2}|S|^{2}+\left\{A m\left(\sum_{i} \frac{\lambda_{N i}}{2} S N_{i}^{2}+\frac{\lambda_{S}}{3 !} S^{3}\right)+\text { H.c. }\right\}\right] .
\end{gathered}
$$

The term $V_{\text {soft }}^{\mathrm{MSSM}}$ represents the MSSM soft terms. (Note that the $B$ term $B \mu H_{1} H_{2}$ is not included.) We have taken the common soft breaking parameters $m$ and $A$ for the singlet fields $N_{i}, S$ in Eq. (6), motivated by the supergravity scenario [11]. That is, if we set the common trilinear coupling $A$ and universal soft mass $m$ at the Planck scale, the $A$ parameters and soft breaking masses composed purely of gauge singlets

\footnotetext{
${ }^{2}$ There might be the cosmological domain-wall problem associated with the spontaneous $Z_{3}$ symmetry breaking. This may be evaded by, e.g., nonrenormalizable terms or inflation [10].
} 
$N_{i}, S$ [namely, the terms inside the square brackets in Eq. (6)] are relatively insensitive to RGE running and so their values at the weak scale are approximately the same as the values at the Planck scale. In contrast, the MSSM soft terms run in the usual way. The term $V_{D}$ in Eq. (4) represents the MSSM $D$ terms whose neutral components may be written as

$$
V_{D}^{\text {neutral }}=\frac{g_{1}^{2}+g_{2}^{2}}{8}\left(-\left|H_{1}^{0}\right|^{2}+\left|H_{2}^{0}\right|^{2}-\sum_{i}\left|\tilde{\nu}_{i}\right|^{2}\right)^{2} .
$$

Note that the Majorana masses of right-handed neutrinos $M_{R}$ are naturally induced by the VEV of $S$ which is around the supersymmetry breaking scale and not near the unification scale. This is because we have imposed the $Z_{3}$ symmetry, which forbids the explicit Majorana mass terms between the right-handed neutrinos, to generate the $\mu$ term of the Higgs fields $H_{1}$ and $H_{2}$ spontaneously by the VEV of $S$. Since the Majorana masses $M_{R}$ are generated at the order of the supersymmetry breaking scale, the Dirac-type Yukawa couplings $f^{\nu}$ must be below $10^{-6}$, which is around the magnitude of electron, up-quark, or down-quark Yukawa couplings, in order to generate tiny neutrino masses $m_{\nu} \leq \mathrm{eV}$.

The VEV's are determined by the minimization of the potential (4). The right-handed sneutrino $N$ may acquire a VEV along with the $S$, i.e., $R$ parity may be spontaneously broken. In this case, the bilinear $R$-parity violating terms of the form $f^{\nu}\langle N\rangle L_{i} H_{2}$ arise as new sources of neutrino masses $m_{\nu}$. Notice that the VEV for the right-handed sneutrino $N$ does not induce spontaneous lepton number violation. In fact, neither lepton number nor any other global symmetry can be assigned to the superpotential (3). ${ }^{3}$ Therefore, an unwanted Nambu-Goldstone boson accompanied with spontaneously broken global symmetry is absent even if either $S$ or $N$ acquires a VEV.

To gain more insight into the superpotential (3), we show the three lepton number restoring limits. The first is the limit $f^{\nu} \rightarrow 0$. We assign $L=0$ to both $S$ and $N_{i}$. This model forms the NMSSM [9] with extra singlet fields $N_{i}$. The second limit is $\lambda_{N} \rightarrow 0$. This is also the NMSSM with right-handed neutrinos whose lepton number assignments are $L=-1$ for $N$ and $L=0$ for $S$. The third is $\lambda_{H}, \lambda_{S} \rightarrow 0$. This model is called the singlet Majoron model, where $L=-1$ for $N_{i}$ and $L=2$ for $S$ [6]. In the singlet Majoron model, lepton number and $R$ parity are broken spontaneously by the VEV's of the $S$ and $N$.

Now we show the mechanism which generates the VEV's for $S$ and $N$. As mentioned in the previous section, it is not very easy to generate the VEV's in the supersymmetric model. We use the mechanism which requires a large $A$ term [8]. Let us briefly review this mechanism by using a simple model. Consider a superpotential

$$
W=\frac{\lambda}{3} \Phi^{3} .
$$

\footnotetext{
${ }^{3}$ The $R$ symmetry can be assigned as the charge $2 / 3$ for all superfields, but it is broken explicitly by the soft supersymmetry breaking terms.
}

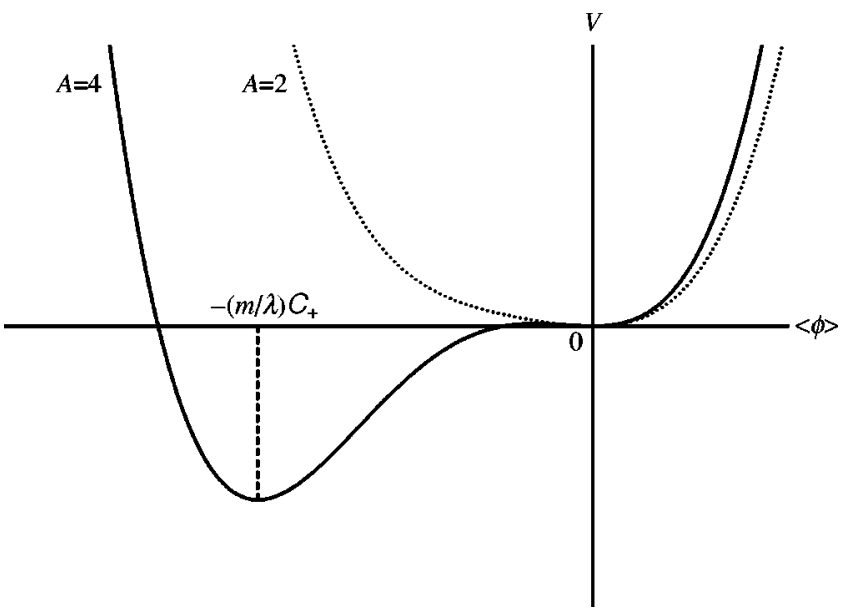

FIG. 1. The schematic figure of the vacuum. The solid line corresponds to $A=4$ and the dotted line corresponds to $A=2$.

Then the scalar potential including the soft terms is given by

$$
V=\left|\lambda \phi^{2}\right|^{2}+\left(\frac{A}{3} m \lambda \phi^{3}+\text { H.c. }\right)+|m|^{2}|\phi|^{2} .
$$

For simplicity, we take $\lambda, A, m$, and $\phi$ to be real. Rescaling the field as $x=(\lambda / m) \phi$, the potential becomes

$$
V=\frac{m^{4}}{\lambda^{2}} x^{2}\left(x^{2}+\frac{2 A}{3} x+1\right) \text {. }
$$

The global minimum is at the origin $x=0$ for $|A|<3$, or at the point $x \neq 0$ for $|A|>3$. When $A>3$, the VEV and potential are given by

$$
\begin{gathered}
\langle\phi\rangle=-\frac{m}{\lambda} C_{+}, \\
V_{\min }=-\frac{m^{4}}{3 \lambda^{2}} C_{+}^{2}\left(C_{+}^{2}-1\right),
\end{gathered}
$$

where

$$
C_{ \pm}=\frac{A \pm \sqrt{A^{2}-8}}{4} \text {. }
$$

This situation is shown in Fig. 1 schematically. It is interesting that both the VEV and the depth of the potential depend on the inverse of $\lambda$. Therefore, we may obtain the larger VEV and the deeper minimum for smaller values of $\lambda$. Originally, efforts have been made to apply this mechanism to the electroweak symmetry breaking of the MSSM in the supergravity scenario, and it has turned out to be difficult [8]. The reason is that the smallest Yukawa coupling $f^{e}, f^{u}$, or $f^{d}$ produces a deeper minimum (where the electric charge is broken) than the desirable one, resulting in the breaking of the electric charge symmetry. In contrast, we apply this mechanism to $R$-parity breaking, leaving the explanation of electroweak symmetry breaking for, say, the radiative breaking. 
Let us analyze the neutral components of the potential (4), and evaluate the VEV for the singlet fields $S$ and $N$. As an illustration, we first treat its simplified version analytically, ignoring the $f^{\nu}$ and $\lambda_{H}$ terms. The $f^{\nu}$ terms can be safely neglected. If we also ignore the $\lambda_{H}$ term, the singlets $S$ and $N_{i}$ decouple from the other fields and the potential is simple enough to be treated analytically. We include $\lambda_{H}$ term as a perturbation later. ${ }^{4}$ The relevant superpotential is given by

$$
W=\frac{\lambda_{N i}}{2} S N_{i}^{2}+\frac{\lambda_{S}}{3 !} S^{3} .
$$

The scalar potential is written as

$$
\begin{aligned}
V= & \sum_{i}\left|\lambda_{N i} S N_{i}\right|^{2}+\left|\sum_{i} \frac{\lambda_{N i}}{2} N_{i}^{2}+\frac{\lambda_{S}}{2} S^{2}\right|^{2}+\sum_{i} m^{2}\left|N_{i}\right|^{2} \\
& +m^{2}|S|^{2}+\left\{A m\left(\sum_{i} \frac{\lambda_{N i}}{2} S N_{i}^{2}+\frac{\lambda_{S}}{3 !} S^{3}\right)+\text { H.c. }\right\} .
\end{aligned}
$$

This potential has the same structure as Eq. (9) and the $S$ and $N$ fields can acquire VEV's if the $A$ terms are large enough.

For simplicity, we do not consider $C P$-violating effects, that is, we assume that all the parameters and VEV's are real. The stationary conditions are given by

$$
\begin{aligned}
\left.\frac{\partial V}{\partial N_{i}}\right|_{\text {vacuum }}= & \lambda_{N i}^{2} s^{2} n_{i}+\left(\sum_{j} \frac{\lambda_{N j}}{2} n_{j}^{2}+\frac{\lambda_{S}}{2} s^{2}\right) \lambda_{N i} n_{i} \\
& +A m \lambda_{N i} s n_{i}+m^{2} n_{i}=0, \\
\left.\frac{\partial V}{\partial S}\right|_{\text {vacuum }}= & \sum_{j} \lambda_{N j}^{2} n_{j}^{2} s+\left(\sum_{j} \frac{\lambda_{N j}}{2} n_{j}^{2}+\frac{\lambda_{S}}{2} s^{2}\right) \lambda_{S} s \\
& +\sum_{j} \frac{A m \lambda_{N j}}{2} n_{j}^{2}+\frac{A m \lambda_{S}}{2} s^{2}+m^{2} s=0,
\end{aligned}
$$

where the VEV's are parametrized as

$$
\langle S\rangle=s,\left\langle N_{i}\right\rangle=n_{i} .
$$

If $n_{i}$ is nonzero, Eq. (16) reduces to

$$
\lambda_{N i}^{2} s^{2}+\lambda_{N i} \xi+m^{2}=0,
$$

where

$$
\xi=\sum_{j} \frac{\lambda_{N j}}{2} n_{j}^{2}+\frac{\lambda_{S}}{2} s^{2}+A m s .
$$

Obviously, Eq. (19) cannot be simultaneously satisfied for all $i=1,2,3$ with nonzero values of $n_{i}$, when $\lambda_{N i}$ are arbitrary. Condition (19) does not change if one includes the $\lambda_{H}$ term. This means that only one of the three $N_{i}$ can acquire a VEV

${ }^{4}$ We solve the full equations (21) and (22) when we calculate the VEV's for $S$ and $N$ numerically in the next section. and the other two VEV's remain zero in our basis. ${ }^{5} \mathrm{We}$ choose parameters such that $n_{3}$ is nonzero. We will write $n_{3}$ and $\lambda_{N 3}$ as $n$ and $\lambda_{N}$, respectively, when it is clear from the context.

Let us classify the global minimum of the scalar potential (15). There are three types depending on the parameters $A$ and $k \equiv \lambda_{S} / \lambda_{N}$. (We treat them in more detail in the Appendix.)

When $|A|<3$, the global minimum is at the origin $n=s$ $=0$. (This corresponds to Solution 1 in the Appendix.) This vacuum is unacceptable, because $s \sim 0$ means that the Higgsino bosons are nearly massless $\mu \sim 0$. In addition, Majorana masses of the right-handed neutrinos also become too small. Therefore the Yukawa couplings $f^{\nu}$ must be extremely small to give proper seesaw masses.

When $|A|>3$, there are two possibilities. One is $n=0, s$ $\neq 0$. (This corresponds to Solution 2 in the Appendix.) This is uninteresting, because in this case the right-handed sneutrino $N$ does not have a VEV, i.e., $R$ parity is unbroken. This amounts to just changing the scales of the ordinary seesaw mechanism. The other is $n \neq 0, s \neq 0$. (This corresponds to Solution 3 in the Appendix.) This can be the global minimum for some range of values of $k$ [that is given by Eq. (A9) in the Appendix]. For example when $|A|=4$, this range corresponds to $1.8 \leq k \leq 6$.

Next, we consider whether the above situation remains the same when we add the $\lambda_{H} S\left(H_{1} H_{2}\right)$ term. Equations (16) and (17) are changed to

$$
\begin{gathered}
\left.\frac{\partial V}{\partial N}\right|_{\text {vacuum }}=\text { Eq. }(16)+\lambda_{H} \lambda_{N} v_{1} v_{2} n=0, \\
\left.\frac{\partial V}{\partial S}\right|_{\text {vacuum }}=\text { Eq. }(17)+\left(\lambda_{H}^{2} v_{2}^{2}+\lambda_{H}^{2} v_{1}^{2}+\lambda_{H} \lambda_{S} v_{1} v_{2}\right) s \\
+A_{H} m \lambda_{H} v_{1} v_{2}=0,
\end{gathered}
$$

where $v_{1}=\left\langle H_{1}^{0}\right\rangle, v_{2}=\left\langle H_{2}^{0}\right\rangle$. The $f^{\nu}$ terms are neglected again. One can see from Eqs. (21) and (22) that the solution corresponding to each case remains the global minimum [for $\left.v_{1}^{2}+v_{2}^{2} \simeq(174 \mathrm{GeV})^{2}\right]$, if $\lambda_{H} \lesssim 1$ and $m \geqslant 100 \mathrm{GeV}$. We have also confirmed this in the numerical calculations.

If one sets $m$ extremely small or adds the term with large $\lambda_{H}(\gtrsim 1)$, the solutions with $s \neq 0$ will not correspond to be the global minimum anymore. The global minimum will then be the unacceptable case $s=0$.

We note that even if we assume common $A$ terms at the Planck scale as in the supergravity scenario, it is easy to make the $A$ parameters for the charged fields small enough $\left(\left|A_{\text {charged }}\right|<3\right)$ to avoid the charge breaking minima, while maintains the $A$ parameters for the neutral fields $S$ and $N$ large enough $(|A|>3)$. This is because only $A_{\text {charged }}$ receives the radiative corrections from gaugino loops running from the Planck to weak scale.

\footnotetext{
${ }^{5}$ There are negligible $O\left(f^{\nu 2}\right)$ corrections once one includes $f^{\nu}$ terms.
} 


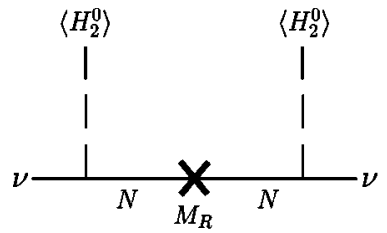

(a)

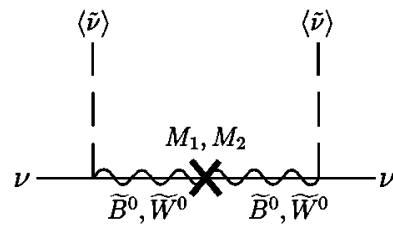

(b)
FIG. 2. The Feynman graph for neutrino masses at tree level. (a) corresponds to the usual seesaw. (b) corresponds to the gaugino seesaw.

\section{THE NEUTRINO MASSES}

We investigate the neutrino masses generated by a seesaw mechanism in which left-handed neutrinos mix with both the right-handed neutrinos and neutralinos. The left-handed sneutrinos acquire VEV's induced by a VEV of the righthanded sneutrino. This is because the nonzero VEV of $N_{3}$ introduces the bilinear $R$-parity violating couplings

$$
f_{i 3}^{\nu} n_{3}\left(L_{i} H_{2}\right) \text {, }
$$

such that the VEV of $H_{2}^{0}$ induces linear terms for the lefthanded sneutrinos [3]. The stationary conditions with respect to left-handed sneutrinos are given by

$$
\begin{aligned}
\left.\frac{\partial V}{\partial \widetilde{\nu_{i}}}\right|_{\text {vacuum }}= & \left(f_{j 3}^{\nu} u_{j} n_{3}+\lambda_{H} s v_{1}\right) f_{i 3}^{\nu} n_{3} \\
& +\left(f_{j 3}^{\nu} u_{j} v_{2}+\lambda_{N} s n_{3}\right) f_{i 3}^{\nu} v_{2}+\sum_{k=1,2}\left(f_{j k}^{\nu} u_{j} v_{2} f_{i k}^{\nu} v_{2}\right) \\
& +\frac{1}{4}\left(g_{1}^{2}+g_{2}^{2}\right) \\
& \times\left(\sum_{j} u_{j}^{2}+v_{1}^{2}-v_{2}^{2}\right) u_{i}+A^{\nu} m f_{i 3}^{\nu} v_{2} n_{3}+m_{L}^{2} u_{i} \\
= & 0,
\end{aligned}
$$

where $u_{i}$ are the VEV's of the left-handed sneutrinos. Ignoring the terms of the second order in $f^{\nu}$, we can easily solve Eq. (24) as

$$
u_{i} \sim-f_{i 3}^{\nu} n_{3}\left\{\frac{\lambda_{H} v_{1} s+\lambda_{N} v_{2} s+A^{\nu} m v_{2}}{\frac{1}{4}\left(g_{1}^{2}+g_{2}^{2}\right)\left(v_{1}^{2}-v_{2}^{2}\right)+m_{L}^{2}}\right\} .
$$

Note that $u$ is $O\left(f^{\nu}\right)$ multiplied by parameters of the order of the weak scale. It is always possible to change the basis of the lepton doublets as

$$
u_{i}^{\prime}=O_{i j} u_{j}
$$

so that only $u_{3}^{\prime}$ takes nonzero value $u_{3}^{\prime}=u$. By this rotation, $f^{\nu}$ changes as

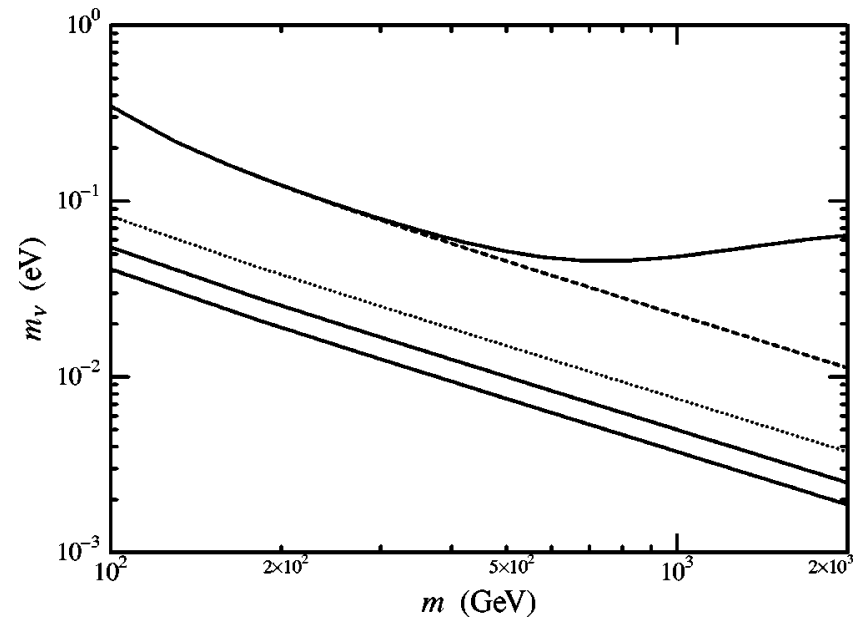

FIG. 3. The three neutrino masses vs the soft scalar mass $m$ of gauge singlets in the case of $n \neq 0$ (corresponding to Solution 3 in the Appendix). The dashed line shows the case of zero gauge couplings, i.e., the case where the gaugino seesaw effect is ignored. The dotted line represents the ordinary seesaw effect between the left- and right-handed neutrinos. The values of the parameters are $f_{1}^{\nu}=f_{2}^{\nu}=f_{3}^{\nu}=5 \times 10^{-7}, \quad \lambda_{H}=0.3, \lambda_{N 1}=0.4, \lambda_{N 2}=0.3, \lambda_{N 3}$ $=0.2, \lambda_{S}=0.6$, and $A=-4$. The gaugino mass values are $M_{2}$ $=2 M_{1}=1 \mathrm{TeV}$.

$$
\begin{aligned}
f_{i j}^{\nu \prime} & =O_{i k} f_{k j}^{\nu} \\
& =\left(\begin{array}{lll}
f_{11}^{\nu \prime} & f_{12}^{\nu \prime} & 0 \\
f_{21}^{\nu \prime} & f_{22}^{\nu \prime} & 0 \\
f_{31}^{\nu \prime} & f_{32}^{\nu \prime} & f_{\nu}
\end{array}\right),
\end{aligned}
$$

where

$$
f_{\nu} \equiv f_{33}^{\nu \prime}=O_{3 k} f_{k 3}^{\nu}=\sqrt{f_{13}^{\nu 2}+f_{23}^{\nu}{ }^{2}+f_{33}^{\nu}{ }^{2}}
$$

Hereafter, we take this base and drop ' from $f^{\nu}$.

In our model, neutrinos mix with neutralinos. The treelevel mass matrix for neutrino-neutralino fields $\left(\nu_{i}, \psi_{N j}, \psi_{S}, \psi_{H_{1}^{0}}, \psi_{H_{2}^{0}}, \widetilde{B}^{0}, \widetilde{W}^{0}\right)$ is given by ${ }^{6}$

$$
\left(\begin{array}{cc}
0_{3 \times 3} & \mathcal{M}_{D} \\
\mathcal{M}_{D}^{T} & \mathcal{M}_{R}
\end{array}\right),
$$

where

\footnotetext{
${ }^{6} \mathrm{We}$ have omitted the mass term $f_{3 j}^{\nu} u_{3} \psi_{N j} \psi_{H_{2}^{0}}$, because this term is second order in $f^{\nu}$ and contributes to the light neutrino masses $m_{\nu}$ at higher orders. (Other terms contribute up to second order.)
} 


$$
\begin{aligned}
\mathcal{M}_{D}= & \left(f_{i j^{\prime}}^{v} v_{2}, 0_{3 \times 1}, 0_{3 \times 1}, \delta_{i 3} f_{\nu} n,-\delta_{i 3} \frac{g_{1} u}{\sqrt{2}}, \delta_{i 3} \frac{g_{2} u}{\sqrt{2}}\right), \\
\mathcal{M}_{R}= & \left(\begin{array}{cccccc}
\delta_{j j^{\prime}} \lambda_{N j^{\prime}} s & \delta_{j 3} \lambda_{N} n & 0_{3 \times 1} & 0_{3 \times 1} & 0_{3 \times 1} & 0_{3 \times 1} \\
\delta_{3 j^{\prime}}, \lambda_{N} n & \lambda_{S} s & \lambda_{H} v_{2} & \lambda_{H} v_{1} & 0 & 0 \\
0_{1 \times 3} & \lambda_{H} v_{2} & 0 & \lambda_{H} s & -g_{1} v_{1} / \sqrt{2} & g_{2} v_{1} / \sqrt{2} \\
0_{1 \times 3} & \lambda_{H} v_{1} & \lambda_{H} s & 0 & g_{1} v_{2} / \sqrt{2} & -g_{2} v_{2} / \sqrt{2} \\
0_{1 \times 3} & 0 & -g_{1} v_{1} / \sqrt{2} & g_{1} v_{2} / \sqrt{2} & M_{1} & 0 \\
0_{1 \times 3} & 0 & g_{2} v_{1} / \sqrt{2} & -g_{2} v_{2} / \sqrt{2} & 0 & M_{2}
\end{array}\right),
\end{aligned}
$$

and $0_{i \times j}$ is $i \times j$ submatrix whose components are all zero. Higgsino mass $\mu$, Majorana masses of right-handed neutrinos $M_{R}$, and Dirac masses $m_{D}$ between left- and right-handed neutrinos are, respectively, given by

$$
\begin{gathered}
\mu \equiv \lambda_{H} s, \\
M_{R i} \equiv \lambda_{N i} s, \\
m_{D i j} \equiv f_{i j}^{\nu} v_{2} .
\end{gathered}
$$

The mass matrix for the three light neutrinos is given by

$$
\begin{aligned}
m_{\nu i j}= & -\mathcal{M}_{D} \mathcal{M}_{R}^{-1} \mathcal{M}_{D}^{T} \\
= & -\sum_{k=1}^{3} \frac{m_{D i k} m_{D j k}}{M_{R k}}-\delta_{i 3} \delta_{j 3}\left[\frac{u^{2}}{2 M}+\frac{f_{\nu} n u v_{1}}{M \mu}\right. \\
& \times\left(1-\frac{\lambda_{H} v^{2} \sin 2 \beta}{X}+\frac{2 \lambda_{H} v_{2}^{2} \tan \beta}{X}\right) \\
& \left.+\frac{\left(f_{\nu} n\right)^{2}}{\mu}\left(\frac{v_{1}^{2}}{2 M \mu}-\frac{\lambda_{H} v^{4} \sin ^{2} 2 \beta}{M \mu X}+\frac{4 \lambda_{H} v_{2}^{2}}{X}\right)\right] \\
& \times\left\{1-\frac{v^{2}}{2 M \mu}\left(\sin 2 \beta+\frac{\lambda_{H} v^{2}}{X} \cos ^{2} 2 \beta\right)\right\}^{-1},
\end{aligned}
$$

where

$$
X \equiv \lambda_{S} s^{2}-\lambda_{N} n^{2}+2 \lambda_{H} v_{1} v_{2}
$$

the "reduced" gaugino mass parameter $M$ is defined by

$$
\frac{1}{M}=\frac{g_{1}^{2}}{M_{1}}+\frac{g_{2}^{2}}{M_{2}}
$$

and $v^{2} \equiv v_{1}^{2}+v_{2}^{2} \simeq(174 \mathrm{GeV})^{2}$. We have taken the parametrization $v_{1}=v \cos \beta, v_{2}=v \sin \beta$ which is justified since $u_{i} \ll v_{1}, v_{2}$.

Before presenting the numerical results, we provide qualitative explanations taking two limits in Eq. (35). The first term $-m_{D i k} m_{D j k} / M_{R k}$ in Eq. (35) comes from the ordinary seesaw effect between left- and right-handed neutrinos as shown in Fig. 2(a). We denote this as "usual seesaw." The second term in Eq. (35) is characteristic of our model.

In the limit $s \rightarrow \infty$ and $v \rightarrow 0$, Eq. (35) reduces to

$$
m_{\nu i j}=-\delta_{i 3} \delta_{j 3} \frac{u^{2}}{2 M}
$$

which is simply the first term in the square bracket in Eq. (35). This comes from the "gaugino seesaw" effect shown in Fig. 2(b). The neutrino mass generation through the gaugino seesaw effect is characteristic of a bilinear $R$-parity violating model [3]. [As mentioned, our model has the effective bilinear $R$-parity violating coupling (23).]

If we take the limit $M \rightarrow \infty$, Eq. (35) becomes

$$
m_{\nu i j}=-\sum_{k=1}^{3} \frac{m_{D i k} m_{D j k}}{M_{R k}}-\delta_{i 3} \delta_{j 3} \frac{\left(f_{\nu} n\right)^{2}}{\mu} \frac{4 \lambda_{H} v_{2}^{2}}{X}
$$

The first term in Eq. (39) is just the usual seesaw mass term. The second term in Eq. (39) comes from the "Higgsino seesaw" effect via the Dirac mass $f_{\nu} n$ which mixes Higgsino $\psi_{H_{2}^{0}}$ and left-handed neutrino of third generation, which originates from the effective bilinear $R$-parity breaking coupling (23). The origin of this second term in Eq. (39) is the last term in the square bracket in Eq. (35). The Higgsino seesaw effect is absent in the bilinear $R$-parity violating model. The difference is that if we rotate $\left(L_{i}, H_{1}\right)$ to eliminate the bilinear terms (23), there appear $\left(L_{i} H_{2}\right) S$ terms which are absent in the bilinear $R$-parity violating model.

The interesting point here is that the neutrino mass matrix (39) is purely given by the usual seesaw effect except for its 3-3 element. The gaugino and Higgsino seesaw together work only for one generation, because we can always rotate the basis as in Eq. (26). Thus the hierarchical neutrino mass structure arises even if the Dirac-type Yukawa couplings $f^{\nu}$ are all the same order of magnitude.

Now we show our numerical result in Fig. 3 using the parameters explained as follows. In the calculation, we treat the VEV's of the Higgs fields $v_{1}$ and $v_{2}$ as inputs such that $v_{1}=v \cos \beta, v_{2}=v \sin \beta$, where $v \simeq 174 \mathrm{GeV}$ and $\tan \beta=10$. 
(This can be realized by solving the stationary conditions of the scalar potential with respect to $H_{1}^{0}$ and $H_{2}^{0}$ for the soft scalar masses $m_{H_{1}}$ and $m_{H_{2}}$.) We search for the global minimum of the potential (4) to obtain the VEV's for $S$ and $N$ using Eqs. (21) and (22), and then evaluate the neutrino masses from the eigenvalues of the mass matrix (29). We input the soft supersymmetry breaking parameters as

$$
\begin{aligned}
A & =-4, \\
m_{L}^{2} & =m^{2}+0.5 M_{0}^{2}, \\
M_{2} & =2 M_{1}=0.8 M_{0}=1 \mathrm{TeV},
\end{aligned}
$$

motivated by the supergravity scenario. (The overall form of Fig. 3 does not change if we set another inputs for soft breaking e.g. $m_{L}^{2}=m^{2}$.) We take the following values for the Yukawa couplings:

$$
\begin{gathered}
\lambda_{N 1}=0.4, \quad \lambda_{N 2}=0.3, \quad \lambda_{N 3}=0.2, \\
\lambda_{H}=0.3, \quad \lambda_{S}=0.6, \\
f_{i j}^{\nu}=\left(\begin{array}{ccc}
1 & 0 & 0 \\
0 & 1 / \sqrt{2} & 1 / \sqrt{2} \\
0 & -1 / \sqrt{2} & 1 / \sqrt{2}
\end{array}\right) \\
\times\left(\begin{array}{ccc}
5 \times 10^{-7} & 0 & 0 \\
0 & 5 \times 10^{-7} & 0 \\
0 & 0 & 5 \times 10^{-7}
\end{array}\right) .
\end{gathered}
$$

The above parameters are chosen such that we obtain the global minimum with spontaneous $R$-parity violation $n \neq 0$ (corresponding to Solution 3 in the Appendix), and that we may account for the solar neutrino by the MSW effect [12] between $\nu_{e}$ and $\nu_{\mu}$ and the atmospheric neutrino by mixing between $\nu_{\mu}$ and $\nu_{\tau}$. With this choice, the mass of the third generation $\sim 5 \times 10^{-2} \mathrm{eV}$ accounts for the atmospheric neutrino oscillations, and the mass of the second generation $\sim 3 \times 10^{-3} \mathrm{eV}$ accounts for the solar neutrino oscillations.

We can see in Fig. 3 that the third generation is substantially heavier than the other two due to the Higgsino and gaugino seesaw effect. The dotted line represents the usual seesaw effect for the third generation $m_{D 3}^{2} / M_{R 3}$. The dashed line represents the Higgsino seesaw effect [the second term in Eq. (39)]. We can see that in the large $m$ region, the gaugino seesaw effect shown in Eq. (38) becomes dominant for the third generation. The reason is that $s$ and $n$ are both proportional to $m$ [see Eq. (A3) in the Appendix]; hence we can show from Eqs. (32) and (33) that the usual and Higgsino seesaw effects (39) are inversely proportional to $m$, so that they are less significant in the large $m$ region.

Let us now discuss the neutrino mixing. The neutrino mixing matrix $U$ diagonalizes the $m_{\nu}$ shown in Eq. (35):

$$
U^{\mathrm{T}} m_{\nu} U=\operatorname{diag}\left(m_{\nu 1}, m_{\nu 2}, m_{\nu 3}\right) .
$$

At first sight, it might seem difficult to maintain large mixing between the second and third generation as required to fit atmospheric neutrino data, because $m_{\nu}$ is hierarchical (namely 3-3 element is largest). However, the observable mixing matrix [13] in the neutrino oscillation experiment is not $U$ but

$$
O^{T} U
$$

where $O$ is the arbitrary mixing matrix appearing in Eq. (27), determined by the free parameters $f_{13}^{\nu}, f_{23}^{\nu}$, and $f_{33}^{\nu}$ in the original basis of Eq. (26). Therefore, we may obtain sufficiently large mixing angle(s) to account for the atmospheric and/or solar neutrino oscillation(s).

\section{SUMMARY}

We have studied the supersymmetric standard model with right-handed neutrinos $N_{i}$ and a singlet field $S$, without assuming extra symmetries such as lepton number. The Majorana masses of the right-handed neutrinos are spontaneously induced around the TeV region by the VEV of the singlet field $S$, which is generated by the effect of the large $A$ term. The right-handed sneutrino $N$ may acquire a VEV as well, leading to spontaneous $R$-parity violation. In this case, the effective theory is similar to a bilinear $R$-parity violating model.

There are two sources for the neutrino masses. One is the "usual seesaw" mechanism between left- and right-handed neutrinos. The other is the bilinear $R$-parity violating effect coming from the terms $f_{i 3}^{\nu} n L_{i} H_{2}$.

The usual seesaw contributes to all the elements of the neutrino mass matrix $m_{\nu i j}$, which generates suitable mass differences for the solar neutrino oscillation due to the MSW effect. The mass difference appropriate for the atmospheric neutrino oscillation is obtained by the bilinear $R$-parity breaking effect which contributes only to the third generation. The hierarchical neutrino mass structure naturally arises even if one sets all the neutrino Yukawa couplings $f^{\nu}$ to be of the same order. We may obtain suitable mixing angles for both the atmospheric and solar neutrinos.

\section{ACKNOWLEDGMENTS}

The authors are most grateful to Y. Okada for discussions, advice, and for reading the manuscript. In addition, we would like to thank A. Akeroyd for reading the manuscript, and T. Goto, J. Hisano, and K. Ishikawa for comments. K.O. thanks the Japan Society for the Promotion of Science for financial support.

\section{APPENDIX: THE VACUA OF THE SIMPLIFIED POTENTIAL}

We investigate the vacua of the scalar potential (15). The solutions of the stationary conditions (16) and (17) may be classified into four types:

Solution 1:

$$
n_{i}=s=0 .
$$


Solution 2:

$$
n_{i}=0, s=-\frac{2 m}{\lambda_{S}} C_{ \pm} .
$$

Solution 3:

$$
n^{2}=\frac{m^{2}}{\lambda_{N}^{2}}\left\{\frac{A^{2} k}{(1+k)^{2}}-2\right\}, s=-\frac{A m}{\lambda_{N}} \frac{1}{1+k} .
$$

Solution 4:

$$
n^{2}=\frac{m^{2}}{\lambda_{N}^{2}} C_{ \pm}^{2}(2-k), s=-\frac{m}{\lambda_{N}} C_{ \pm},
$$

where $k=\lambda_{S} / \lambda_{N}$ (note that $k>0$ in our base). The value of the potential (15) at each solution is, respectively,

$$
\begin{gathered}
V_{1}=0, \\
V_{2}^{ \pm}=-\frac{4}{3} \frac{m^{4}}{\lambda_{S}^{2}} C_{ \pm}^{2}\left(C_{ \pm}^{2}-1\right), \\
V_{3}=-\frac{m^{4}}{\lambda_{N}^{2}}\left\{\frac{A^{4} k}{3(k+1)^{3}}-\frac{A^{2}}{k+1}+1\right\}, \\
V_{4}^{ \pm}=-\frac{m^{4}}{\lambda_{N}^{2}} C_{ \pm}^{2}\left(C_{ \pm}^{2}-1\right)\left(1-\frac{k}{3}\right) .
\end{gathered}
$$

When $|A|<3$, solution 1 becomes the global minimum of the potential (15). When $|A|>3$, Solution 3 becomes the global minimum if

$$
\frac{1}{6 C_{+}^{2}}\left(2+2 C_{+}^{2}+\frac{8 \cdot 2^{1 / 3} C_{+}^{4}}{f}+2^{1 / 3} f\right)<k<2 C_{+}^{2},
$$

where

$$
\begin{aligned}
f= & \left\{-1-3 C_{+}^{2}+20 C_{+}^{6}+\left(1+2 C_{+}^{2}\right)\right. \\
& \left.\times \sqrt{1+2 C_{+}^{2}-3 C_{+}^{4}-36 C_{+}^{6}+36 C_{+}^{8}}\right\}^{1 / 3},
\end{aligned}
$$

otherwise Solution 2 becomes the global minimum. There is no region where Solution 4 becomes the global minimum. The reason is as follows. We look for the parameter region that realizes $V_{4}<V_{1}, V_{2}, V_{3}$. To have $V_{4}<V_{1}$ and $n^{2}>0$, we need $C_{ \pm}^{2}-1>0$ (which is equivalent to $|A|>3$ ). Using $C_{ \pm}^{2}$ $-1>0$, the condition $V_{4}<V_{2}$ reads $(k-2)^{2}(k+1)<0$. This cannot be maintained for all $k>0$. That is, Solution 4 is not global minimum.

Let us derive the condition (A9) for Solution 3. We consider the case $A>0$ because the extension to the $A<0$ case is trivial. The condition for $n^{2}>0$ is

$$
\begin{gathered}
2 \sqrt{2}<A, \\
2 C_{-}^{2}<k<2 C_{+}^{2} .
\end{gathered}
$$

The global minimum condition is given by

$$
\begin{gathered}
V_{3}<V_{1} \text { when } 2 \sqrt{2}<A<3, \\
V_{3}<V_{2} \text { when } 3<A .
\end{gathered}
$$

When $A<3$ we rewrite the condition (A13) using Eqs. (A5) and (A7) such that

$$
k>\frac{1}{3}\left\{A^{2}-3+\left(9 A^{4}-A^{6}\right)^{1 / 3}\right\}>2 C_{+}^{2},
$$

which is inconsistent with Eq. (A12). Therefore, it is not Solution 3 but Solution 1 which represents the global minimum of the potential in the case of $A<3$. When $A>3$, condition (A9) follows from Eqs. (A6), (A7), and (A14). Condition (A9) is compatible with condition (A12), and we may find nonzero solutions (A3) for $n$ and $s$. As mentioned below Eq. (19), only one of the three $N_{i}$ may acquire a VEV. We isolate it by comparing the value of $V_{3}$ for each $\lambda_{N i}$. $\left(V_{3}\right.$ takes its minimum value when $k=A-1$. Therefore, if $k_{1}, k_{2}, k_{3}>|A|-1$, the right-handed sneutrino that acquires the VEV is the one with the smallest $\lambda_{N i}$.)
[1] Super-Kamiokande Collaboration, Y. Fukuda et al., Phys. Rev. Lett. 81, 1562 (1998); Super-Kamiokande Collaboration, M. Nakahata, Nucl. Phys. B (Proc. Suppl.) 76, 425 (1999).

[2] T. Yanagida, in Proceedings of the Workshop on Unified Theory and Baryon Number of the Universe, edited by $\mathrm{O}$. Sawada and A. Sugamoto (KEK, Tokyo, 1979), p. 95; M. Gell-Mann, P. Ramond, and R. Slanski, in Supergravity, edited by P. van Nieuwenhuizen and D. Freedman (North-Holland, Amsterdam, 1979).

[3] L.J. Hall and M. Suzuki, Nucl. Phys. B231, 419 (1984); T. Banks, Y. Grossman, E. Nardi, and Y. Nir, Phys. Rev. D 52, 5319 (1995); H. Nilles and N. Polonsky, Nucl. Phys. B484, 33
(1997); E. Nardi, Phys. Rev. D 55, 5772 (1997); S. Roy and B. Mukhopadhyaya, ibid. 55, 7020 (1997); R. Hempfling, hep-ph/9702412; M.A. Diaz, J.C. Romao, and J.W. Valle, Nucl. Phys. B524, 23 (1998); E.J. Chun, S.K. Kang, C.W. Kim, and U.W. Lee, ibid. B544, 89 (1999); V. Bednyakov, A. Faessler, and S. Kovalenko, Phys. Lett. B 442, 203 (1998); J.W. Valle, hep-ph/9808292; J. Ferrandis, Phys. Rev. D 60, 095012 (1999); D.E. Kaplan and A.E. Nelson, J. High. Energy Phys. 01, 033 (2000); C. Chang and T. Feng, Eur. Phys. J. C 12, 137 (2000); A. Datta, B. Mukhopadhyaya, and S. Roy, Phys. Rev. D 61, 055006 (2000); J.C. Romao, hep-ph/9907466; J.C. Romao, M.A. Diaz, M. Hirsch, W. Porod, and J.W. Valle, Phys. Rev. D (to be published), 
hep-ph/9907499; M. Hirsch, hep-ph/9909443; F. Takayama and M. Yamaguchi, hep-ph/9910320.

[4] For review, see H.P. Nilles, Phys. Rep. 110, 1 (1984).

[5] A. Santamaria and J.W. Valle, Phys. Lett. B 195, 423 (1987); A. Masiero and J.W. Valle, ibid. 251, 273 (1990); D. Comelli, A. Masiero, M. Pietroni, and A. Riotto, ibid. 324, 397 (1994).

[6] Y. Chikashige, R.N. Mohapatra, and R.D. Peccei, Phys. Lett. 98B, 265 (1981); G. F. Giudice, A. Masiero, M. Pietroni, and A. Riotto, Nucl. Phys. B396, 243 (1993); J.M. Cline, K. Kainulainen, and S. Paban, Phys. Lett. B 319, 513 (1993); I. Umemura and K. Yamamoto, Nucl. Phys. B423, 405 (1994); J. Sirkka and I. Vilja, Phys. Lett. B 332, 141 (1994).

[7] K. Inoue, A. Kakuto, H. Komatsu, and S. Takeshita, Prog. Theor. Phys. 67, 1889 (1982); 68, 927 (1982); 71, 413 (1984); L. Alvarez-Gaumé, J. Polchinski, and M.B. Wise, Nucl. Phys. B221, 495 (1983); K. Inoue, A. Kakuto, and S. Takeshita, Prog. Theor. Phys. 71, 348 (1984).

[8] H.P. Nilles, M. Srednicki, and D. Wyler, Phys. Lett. 120B, 346 (1983); R. Gatto and G. Sartori, ibid. 124B, 333 (1983); J.M.
Frere, D.R. Jones, and S. Raby, Nucl. Phys. B222, 11 (1983).

[9] P. Fayet, Nucl. Phys. B90, 104 (1975); R. Barbieri, S. Ferrara, and C.A. Savoy, Phys. Lett. 119B, 343 (1982); J.P. Derendinger and C.A. Savoy, Nucl. Phys. B237, 307 (1984); J. Ellis, J.F. Gunion, H.E. Haber, L. Roszkowski, and F. Zwirner, Phys. Rev. D 39, 844 (1989).

[10] B. Holdom, Phys. Rev. D 28, 1419 (1983); B. Rai and G. Senjanovic, ibid. 49, 2729 (1994); S.A. Abel, S. Sarkar, and P.L. White, Nucl. Phys. B454, 663 (1995).

[11] A.H. Chamseddine, R. Arnowitt, and P. Nath, Phys. Rev. Lett. 49, 970 (1982); R. Barbieri, S. Ferrara, and C.A. Savoy, Phys. Lett. 119B, 343 (1982); L. Hall, J. Lykken, and S. Weinberg, Phys. Rev. D 27, 2359 (1983).

[12] L. Wolfenstein, Phys. Rev. D 17, 2369 (1978); S.P. Mikheev and A.Y. Smirnov, Yad. Fiz. 42, 1441 (1985) [Sov. J. Nucl. Phys. 42, 913 (1985)]; Nuovo Cimento C 9C, 17 (1986).

[13] Z. Maki, M. Nakagawa, and S. Sakata, Prog. Theor. Phys. 28, 870 (1962). 